[Article]

\title{
基于金银合金薄膜的波长检测型表面等离子体共振传感器
}

\author{
张 喆 ${ }^{1}$ 刘 杰 ${ }^{1, *}$ 逯丹凤 ${ }^{2}$ 祁志美 ${ }^{2}$ \\ ( ${ }^{1}$ 北京交通大学计算机与信息技术学院, 生物医学工程系, 北京 100044 ; \\ 2 中国科学院电子学研究所, 传感技术联合国家重点实验室, 北京 100190)
}

\begin{abstract}
摘要: 从实验和理论两方面详细研究了金银合金膜表面等离子体共振(SPR)传感器在可见光波段的敏感特 性. 实验方面, 通过在玻璃基底上浌射 $50 \mathrm{~nm}$ 厚的金银合金薄膜制备了一种新型的 SPR 传感芯片, 并且自行搭 建了基于Kretschmann 结构的波长检测型 SPR 传感器测试平台. 利用不同浓度的氯化钠 $(\mathrm{NaCl})$ 水溶液和浓度 为 $10 \mu \mathrm{mol} \cdot \mathrm{L}^{-1}$ 的牛血清蛋白(BSA)水溶液分别作为折射率样品和分子吸附样品, 研究了传感器的折射率灵敏 度和吸附灵敏度, 并与金膜和银膜 SPR 传感器进行了对比研究. 结果表明, 对于折射率灵敏度的测试, 金银合 金膜 SPR 传感器大幅高于金膜 SPR 传感器, 略低于银膜 SPR 传感器; 而对于吸附敏感的研究, 金银合金膜 SPR 传感器的灵敏度与银膜 SPR 传感器相近, 是金膜 SPR 传感器的 3 倍. 理论方面, 利用菲涅尔公式和等效折 射率计算公式仿真计算了这三种薄膜结构的 SPR 传感器的灵敏度和精确度, 结果指出金银合金膜 SPR 传感器 的灵敏度与银膜 SPR 传感器接近, 是常规金膜 SPR 传感器的 2.31 倍, 而半高峰宽仅为金膜和银膜 SPR 传感器 的 1.36 倍. 在稳定性方面, 金银合金膜 SPR 传感器与金膜 SPR 传感器均具有良好的化学稳定性, 而银膜 SPR 传感器较易氧化, 使用寿命低, 不常被采用. 综上, 金银合金膜在改善传感器灵敏度的同时, 不会降低精度, 是一 种高灵敏、低成本、良好稳定性的SPR传感器敏感材料.
\end{abstract}

关键词：金银合金薄膜；表面等离子体共振；波长检测型；灵敏度

中图分类号: 0649

\section{Wavelength-Interrogated Surface Plasmon Resonance Sensor Based on Au-Ag Alloy Film}

\author{
ZHANG Zhe ${ }^{1} \quad$ LIU Jie ${ }^{1, *} \quad$ LU Dan-Feng ${ }^{2} \quad$ QI Zhi-Mei \\ ('Department of Biomedical Engineering, School of Computer and Information Technology, Beijing Jiaotong University, \\ Beijing 100044, P. R. China; ${ }^{2}$ State Key Laboratory of Transducer Technology, Institute of Electronics, \\ Chinese Academy of Sciences, Beijing 100190, P. R. China)
}

\begin{abstract}
An Au-Ag alloy film surface plasmon resonance (SPR) sensor was studied experimentally and theoretically. SPR chips were prepared by sputtering a glass surface with a 50-nm-thick Au-Ag alloy film, and experiments were carried out with the wavelength-interrogated SPR sensor using the Kretschmann configuration. Aqueous sodium chloride and bovine serum albumin solutions were used to study the refractive index and adsorption sensitivities of the sensor. The results were compared with those obtained using Au film and Ag film SPR sensors. The refractive index sensitivity of the Au-Ag alloy film SPR sensor is higher than that of the Au film SPR sensor, but lower than that of the Ag film SPR sensor. The adsorption sensitivity of the AuAg alloy film SPR sensor is similar to that of the Ag film SPR, and three times of the Au film SPR sensor. Theoretical studies showed that that the sensitivity of the Au-Ag alloy film SPR is close to that of the Ag film SPR sensor, and 2.31 times of the Au film SPR sensor. The full width at half maximum of the Au-Ag film sensor is
\end{abstract}

Received: May 13, 2014; Revised: July 7, 2014; Published on Wed: July 7, 2014.

"Corresponding author. Email: jieliu@bjtu.edu.cn.

The project was supported by the Fundamental Research Funds for the Central Universities, China (2014JBM026).

中央高校基本科研业务费(2014JBM026)资助项目

(c) Editorial office of Acta Physico-Chimica Sinica 
only 0.36 times of the Au or Ag film SPR sensors. The Au-Ag alloy film and Au film SPR sensors are chemically stable, but the Ag film SPR sensor is easily oxidized, so it is not often used. These results show that an Au-Ag alloy film can improve the sensitivity of the sensor, while retaining the accuracy. Au-Ag films could therefore be used as high-sensitivity, low cost, and stable SPR-sensitive materials.

Key Words: Au-Ag alloy film; Surface plasmon resonance; Wavelength interrogation; Sensitivity

\section{1 引言}

表面等离子体共振(SPR)作为一种免标记的在 线监测技术, 可以原位实时观测生物分子相互作用 的过程, 已经广泛应用于生物医学、蛋白质组学、药 物篮选、遗传物质检测、免疫调节、病毒监测以及医 学影像等领域..$^{-16}$ 当入射的 $p$ 偏振光通过棱镜耦合 照射在金属与介质界面并发生全反射时会产生消 逝场, 它对于表界面折射率的变化非常敏感, 因此 当穿过金属层的消逝场与待检测物相互作用时, 由 于分子吸附在金属表面或待检测物体折射率的变 化都会导致传感器检测信号发生改变, 从而实现对 生化物质的检测.

目前常规 SPR 传感芯片的金属敏感膜主要是 金膜, 它具有化学稳定性好的优点, 但是价格昂贵 且灵敏度不高. ${ }^{17}$ 银膜 SPR 传感器在不考虑其易氧 化的缺点下, 理论计算得到的灵敏度结果大幅高于 金膜 SPR 传感器, 但是在实际使用中却受限于它的 化学不稳定性而不常被采用. ${ }^{18,19}$ 为了解决常规金膜 SPR 传感器灵敏度低, 银膜易氧化的问题, 在前期工 作 ${ }^{20}$ 中已经提出采用金银合金膜取代常规的金膜作 为 SPR 传感器的敏感膜, 并且研究了基于金银合金 膜 SPR 传感器在近红外波段的传感特性, 取得了较 好的实验结果.

在本文中, 我们采用自建的基于 Kretschmann 棱镜耦合模型的波长检测型 SPR 传感器, 在可见光 波段对基于金银合金膜、金膜以及银膜等三种金属 膜结构的 SPR 传感器的敏感特性进行了深入研究. 采用不同浓度的氯化钠水溶液和浓度为 $10 \mu \mathrm{mol} \cdot \mathrm{L}^{-1}$ 的牛血清蛋白水溶液分别作为折射率和吸附灵敏 度实验的样品溶液, 依据菲涅耳公式和等效折射率 方程从理论和实验两方面对这三种传感器的灵敏 度、精确度等参数进行对比研究. 理论与实验结果 均表明金银合金膜 SPR 传感器在可见光波段的灵 敏度明显优于常规金膜 SPR 传感器, 达到了与银膜 SPR 传感器相近的水平. 此外, 金银合金膜 SPR 传感 器的检测精确度比金膜或银膜 SPR 传感器略低, 但 对共振波长的确认影响不大.

\section{2 实验部分}

\section{1 仪器与试剂}

HR4000 型电荷耦合器件 (CCD) 光谱分析仪、 LS-1 型卤铇灯 (美国 Ocean Optics 公司); 玻璃基片 (厚度为 $0.8 \mathrm{~mm}$, 日本 Matsunami 会社); 硅橡胶测试 槽 (日本); 透镜、线性偏振片 (北京大恒光电技术公 司); 等腰直角玻璃棱镜(北京北东光电自动化开发 公司); 多模石英光纤(浙江雷畴科技有限公司); 蠕 动泵(保定兰格恒流洜有限公司); 数字阿贝折射仪 (上海光学仪器研究所).

氯化钠 $(\mathrm{NaCl}$, 分析纯, 相对分子质量 58.44$)$ 购 自国药集团化学有限公司; 牛血清蛋白(BSA, 未经 二次提纯, 相对分子质量 66000), 购自美国 SigmaAldrich 公司. 溶液配制使用经 Milli-Q 纯净水机二 次净化的去离子水.

\section{2 实验装置}

\subsection{1 芯片制备及仪器搭建}

本文所使用的三种 SPR 芯片均采用真空溅射法 制备而得: 首先将作为基底的玻璃基片进行超声清 洗, 之后依次在其表面溅射 $3 \mathrm{~nm}$ 厚的铬膜和 $50 \mathrm{~nm}$ 厚的金银合金膜(或纯金膜、纯银膜)作为敏感膜, 其 中用于溅射金银合金膜的靶材中两组分的质量比为 1:1. 在玻璃基片上溅射了敏感金属膜后即制备成本 文所使用的 SPR 芯片. 图 1 所示为金银合金膜的 X射 线衍射 $(X R D)$ 谱, 扫描范围为 $30^{\circ}-80^{\circ}$, 扫描步长为 $0.001^{\circ}$. 图 1 中所示的三个衍射峰角度分别为: $38.2^{\circ}$ 、 $41.5^{\circ} 、 77.8^{\circ}$, 通过与文献 ${ }^{21}$ 对照可知, 其对应的晶面 为: (111)、(200)、(311), 且(111)面的强度值最大, 该结 果表明溅射法制备的金银合金膜是高度结晶的.

将制备好的 SPR 芯片固定在图 2 所示的基于波 长检测型的 SPR 传感器测量平台上, SPR 芯片的玻 璃基底一面紧贴在棱镜底面上, 另一面的敏感膜暴 露在测试槽内. 卤铇灯发出的多色光穿过多模石英 光纤、聚焦透镜及线性偏振片后变为 $p$ 偏振的准平 行光束 (发散角小于 $0.2^{\circ}$ ), 然后以图 2 所示的角度 $\theta$ 照射到玻璃棱镜上. 进入棱镜的光束在 SPR 芯片的 玻璃基底/敏感膜界面发生全反射(全反射角为 $\varphi$ ), 


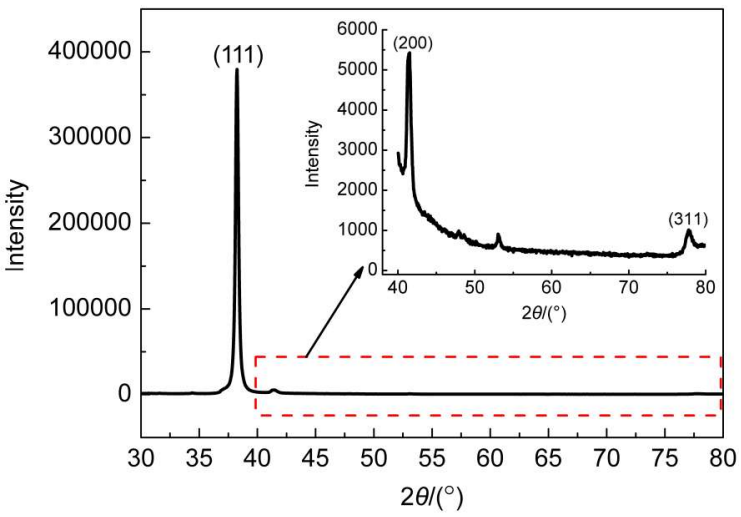

图 1 金银合金溅射膜的 X 射线衍射(XRD)

Fig.1 X-ray diffraction (XRD) patterns of the sputtered Au-Ag alloy film

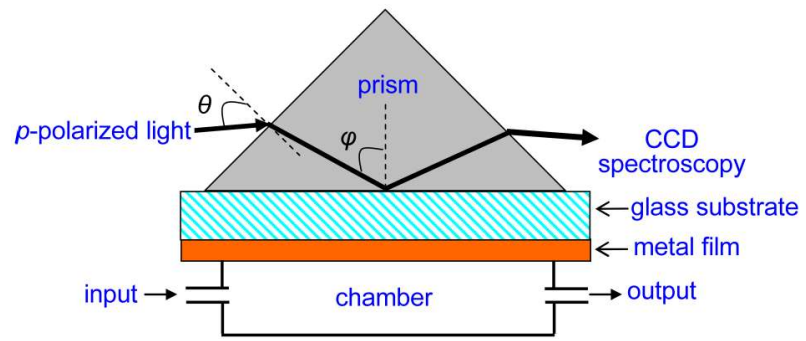

图 2 基于 Kretschmann 结构的波长检测型 SPR 传感器示意图

Fig.2 Schematic diagram of a wavelength-interrogated SPR sensor with Kretschmann configuration

从棱镜另一端面输出的全反射光束被透镜聚焦后 经光纤传输到 $\mathrm{CCD}$ 光谱仪中实时记录, 并传输到计 算机进行处理. 本论文的实验数据均是利用该传感 装置测量取得, 所使用的光谱仪的波长分辨率为 $0.2 \mathrm{~nm}$, 光谱窗口范围为 $200-1100 \mathrm{~nm}$.

\subsection{2 实验方法}

实验采用 $\mathrm{NaCl}$ 水溶液用于折射率灵敏度测量, 其质量百分比浓度为 $0 \% 、 2 \% 、 4 \% 、 6 \% 、 8 \% 、 10 \%$, 阿 贝折射仪测得的相应折射率 $(n)$ 分别为 1.3331 、 $1.3364 、 1.3401 、 1.3436 、 1.3472 、 1.3507$, 与文献值 ${ }^{22}$ 十 分接近. 采用 BSA 水溶液用于蛋白质分子吸附特性 测量, 其浓度为 $10 \mu \mathrm{mol} \cdot \mathrm{L}^{-1}$, 测得的折射率与去离 子水相同均为 1.3331 . 所有测试均在室温 $\left(23^{\circ} \mathrm{C}\right)$ 条 件下进行, 所用样品溶液均由去离子水配制.

在实际测试中, 直接测量的共振反射光谱的半 高峰宽(FWHM)通常较宽, 共振峰不尖锐, 光谱的平 滑度较差, 如图 3 中蓝色曲线所示, 因此不便于精确 确定波谷所对应的共振波长 $\lambda_{\mathrm{R}}$ 的位置. 此时, 我们采 用如下的共振吸收法测试: (1) 记录 CCD 光谱仪的 背景信号强度谱 $I_{\mathrm{B}} ;$; (2) 记录参比物空气的共振反射
光谱 $I_{\mathrm{R}} ;(3)$ 泵出参比物后将样品注入测试槽, 再次 记录共振反射光谱 $I_{\mathrm{s}}$; (4) 通过式(1)计算得到共振吸 收光谱的吸光度 $A$, 共振峰波峰对应的波长即为 $\lambda_{\mathrm{R}}$.

$$
A(\lambda)=-\left[\lg \frac{I_{\mathrm{S}}(\lambda)-I_{\mathrm{B}}(\lambda)}{I_{\mathrm{R}}(\lambda)-I_{\mathrm{B}}(\lambda)}\right]
$$

图 3 中红色曲线是对去离子水测量并计算得到 的共振吸收光谱, 以在此实验条件下不会激发共振 吸收的空气(黑色曲线对应的光谱)作为参比物. 对 比发现对去离子水直接测量得到的强度谱与计算 得到吸收谱确定的 $\lambda_{R}$ 是一致的, 而采用共振吸收方 法对共振峰进行了一定程度的整形和放大, 减小了 光谱的半高峰宽, 因此易于共振峰位置的精确确定.

\section{3 结果与讨论}

\section{1 基于三种贵金属薄膜的 SPR 传感器仿真分析}

图 4 所示为利用 WOOLLAM 椭偏仪测量得到 的金银合金膜、金膜以及银膜的折射率色散曲线, 实线为两种材料折射率的实部 $n$, 虚线为折射率的 虚部 $k$. 图 4 显示金银合金膜和银膜的折射率色散曲 线的 $n 、 k$ 值相近, 与金膜的 $n 、 k$ 值有较大区别, 这是 导致三种材料灵敏度和精确度差异的根本因素, 在 下文的仿真计算结果中会有体现.

图 5(a) 是采用菲涅尔公式 ${ }^{23}$ 计算得到的基于金 银合金膜、金膜和银膜的 SPR 传感器的共振反射强 度谱. $p$ 偏振态四层结构(棱镜/玻璃基底/金属敏感 膜/(测试样品)的菲涅尔反射率公式如下:

$$
R=\left|\frac{r_{12}+r_{234} \mathrm{e}^{-2 a_{2} d_{2}}}{1+r_{12} r_{234} \mathrm{e}^{-2 a_{2} d_{2}}}\right|^{2}
$$

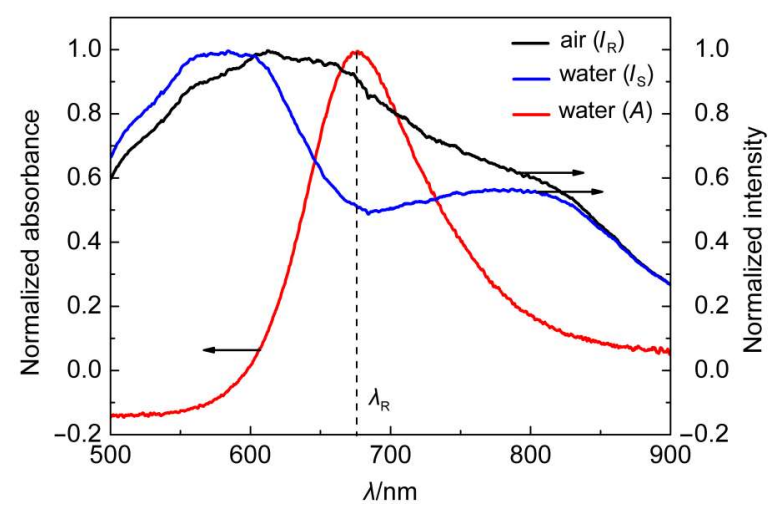

图 3 金银合金膜 SPR 传感器对去离子水测得的共振反射 光谱 $I_{\mathrm{S}}$ 和共振吸收光谱 $A$ (空气作为参照物 $I_{\mathrm{R}}$ )

Fig.3 Resonance reflection $I_{\mathrm{s}}$ and absorbance spectra $A$ of SPR sensor based on the Au-Ag alloy film measured with deionization water (air as the reference $I_{R}$ ) 
其中, $\left\{\begin{array}{l}\alpha_{j}=\left(\beta^{2}-k_{0}^{2} \varepsilon_{j}\right)^{1 / 2}, j=1,2,3,4 \\ \beta=k_{0} \sqrt{\varepsilon_{1}} \sin \varphi \\ r_{234}=\frac{r_{23}+r_{34} \mathrm{e}^{-2 a_{3} d_{3}}}{1+r_{23} r_{34} \mathrm{e}^{-2 a_{3} d_{3}}} \\ r_{j, j+1}=\frac{\varepsilon_{j} \alpha_{j+1}-\varepsilon_{j+1} \alpha_{j}}{\varepsilon_{j} \alpha_{j+1}+\varepsilon_{j+1} \alpha_{j}}\end{array}\right.$

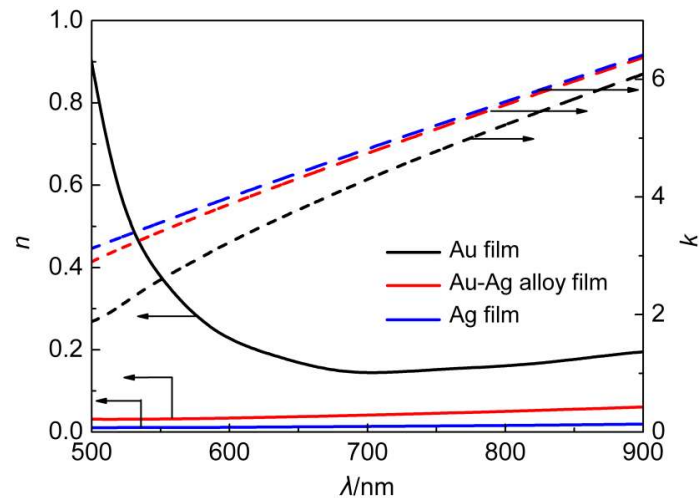

图 4 金银合金膜、金膜以及银膜的折射率色散曲线

Fig.4 Refractive index dispersion curves for the Au-Ag alloy film, Au film, and Ag film
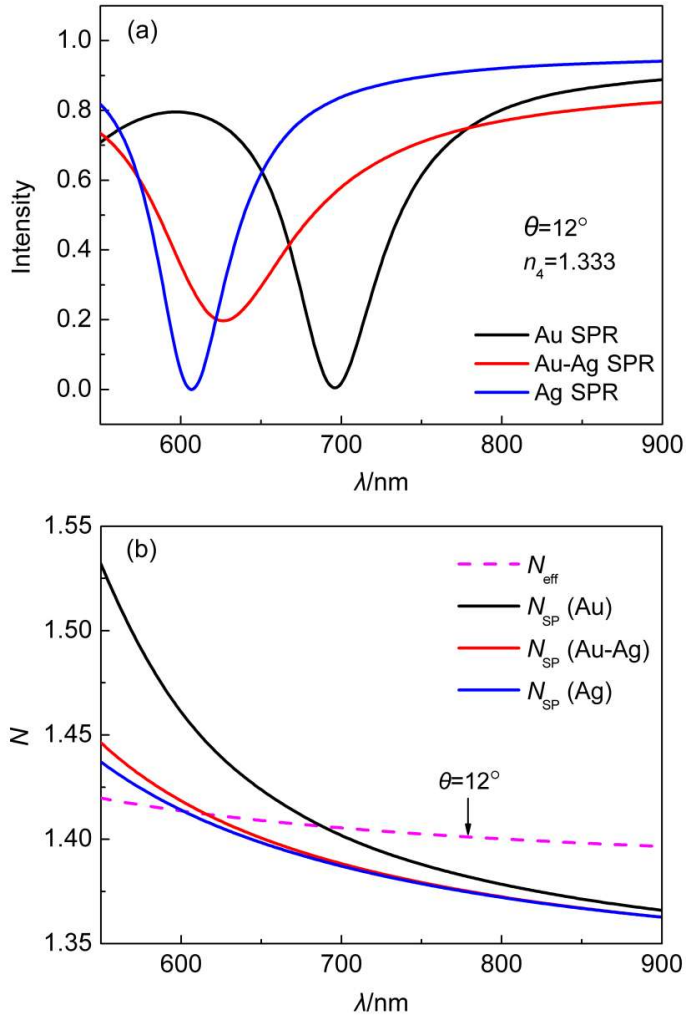

图 5 (a)采用菲涅尔公式计算得到的 SPR 传感器的共振反 射光谱 $\left(\theta=12^{\circ}\right),(\mathrm{b})$ 采用波导本征方程计算得到的金属 膜的等效折射率 $\left(N_{\mathrm{SP}}\right)$ 与入射光波的等效折射率 $\left(N_{\mathrm{eff}}\right)$ 的色散曲线

Fig.5 (a) Resonance reflection spectra of SPR sensor based on Fresnel equation $\left(\theta=12^{\circ}\right)$, (b) effective refractive index dispersions for metal film based on eigenvalue equation of waveguide $\left(N_{\mathrm{SP}}\right)$ and for incident light $\left(N_{\text {eff }}\right)$
上式中 $r_{j, j+1}$ 为 $j$ 层与 $j+1$ 层界面的反射系数, $j=1$ 为 棱镜层, 之后依次为玻璃基底层、金属层及测试样 品层, $d$ 是各层的厚度, $\varepsilon=(n+k \mathrm{i})^{2}$ 是各层的介电常数, 且设定测试样品的折射率为 $n_{4}=1.333$.

从图中可以看出, 对于相同的入射角 $\theta$, 三种材 料计算所得的共振反射光谱都会在一个特定的波 段出现波谷, 表明在该条件下入射光与表面等离子 体波在传播方向上的波矢恰好匹配, 从而激发了表 面等子体共振, 波谷对应的波长就是共振波长 $\lambda_{\mathrm{R}}$. 比 较三种材料的 $\lambda_{\mathrm{R}}$ 可得如下结论: 在入射角度 $\theta$ 相同的 条件下, 基于银膜的 SPR 传感器的 $\lambda_{\mathrm{R}}$ 最小为 607 $\mathrm{nm}$, 基于金银合金膜的 SPR 传感器次之为 $627 \mathrm{~nm}$, 而基于金膜的 SPR 的 $\lambda_{\mathrm{R}}$ 最大为 $696 \mathrm{~nm}$, 三个 $\lambda_{\mathrm{R}}$ 的最 大差别将近 $90 \mathrm{~nm}$.

图 5(b)为依据公式(3)、(4) $)^{20}$ 对上述三种金属敏 感膜计算得到的表面等离子体波的等效折射率 $N_{\mathrm{SP}}$
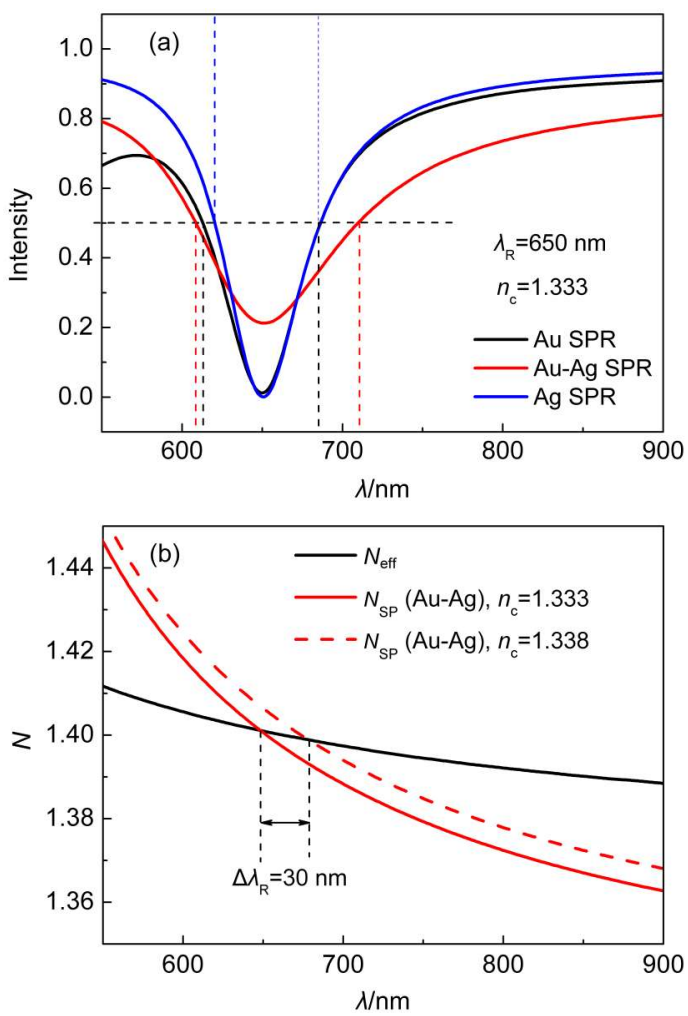

图 6 (a)采用菲涅尔公式计算得到的 SPR 传感器的共振反 射光谱 $\left(\lambda_{R}=650 \mathrm{~nm}\right)$, (b)采用波导本征方程计算得到的金属 膜的等效折射率与入射光波的等效折射率的色散曲线

Fig.6 (a) Resonance reflection spectra of SPR sensor based Fresnel equation $\left(\lambda_{R}=650 \mathrm{~nm}\right)$, (b) effective refractive index dispersions for metal film based eigenvalue equation of waveguide and for incident light

$\lambda_{R}$ : the resonance wavelength corresponding to the three wave trough; $\Delta \lambda_{\mathrm{R}}$ : change of resonance wavelength; $n_{\mathrm{c}}$ : the given refractive index of the sample 
表 1 基于三种金属膜的 SPR 传感器的共振波长、半高峰 宽以及灵敏度对比结果

Table 1 Comparisons of resonance wavelengths, FWHM, and sensitivities of SPR sensors based on three different metal films

\begin{tabular}{lccc}
\hline & $\lambda_{\mathrm{R}} / \mathrm{nm}^{\mathrm{a}}$ & FWHM $/ \mathrm{nm}^{\mathrm{b}}$ & $\Delta \lambda_{\mathrm{R}} / \mathrm{nm}^{\mathrm{c}}$ \\
\hline Au-Ag SPR & 627 & 101 & 30 \\
Au SPR & 696 & 74 & 13 \\
Ag SPR & 607 & 67 & 35 \\
\hline \multicolumn{4}{c}{${ }^{\mathrm{a}} \theta=12^{\circ} ;{ }^{\mathrm{b}} \lambda_{\mathrm{R}}=650 \mathrm{~nm} ;{ }^{\text {c }}$ initial resonance wavelength } \\
$\left(\lambda_{\mathrm{R} 0}\right)=650 \mathrm{~nm}$, change of refractive index $\left(\Delta n_{\mathrm{c}}\right)=0.005 ;$ \\
FWHM: full width at half maximum
\end{tabular}

与通过棱镜耦合的入射光波的等效折射率 $N_{\text {eff }}$ 的色 散关系, 仍然设定测试样品的折射率为 $n_{4}=1.333$.

$$
\begin{aligned}
& N_{\mathrm{SP}}=\sqrt{\frac{\varepsilon_{1}(\lambda) \varepsilon_{2}(\lambda)}{\varepsilon_{1}(\lambda)+\varepsilon_{2}(\lambda)}} \\
& N_{\text {eff }}=n_{1}(\lambda) \sin \varphi
\end{aligned}
$$

其中, $\varphi=\frac{\pi}{4}+\arcsin \left(\frac{\sin \theta}{n_{1}(\lambda)}\right)$, 当 $N_{\mathrm{eff}}=N_{\mathrm{SP}}$, 即曲线相交 时, 达到共振匹配, 此时交点对应的横坐标的波长 即为 $\lambda_{\mathrm{R}}$. 观察图 5(a) 和图 5(b), 两者所确定的 $\lambda_{R}$ 一致, 这三组数据被记录在表 1 中.

设定测试样品折射率 $n_{4}=1.333$ 不变, 通过调节 入射角 $\theta$, 使得基于三种金属膜的 SPR 传感器的共 振波长均位于 $650 \mathrm{~nm}$ 处, 计算结果如图 6(a) 所示. 测量三条曲线各自的 FWHM, 其结果如表 1 所示, 金膜和银膜 SPR 传感器的 FWHM 值接近, 金银合金 膜 SPR 传感器的 FWHM 值略大, 约为金膜的 1.36 倍. FWHM 是影响传感器精确度的一个重要因素, 由此可见基于金银合金膜的 SPR 传感器的检测精 确度较金膜或者银膜 SPR 传感器的略低.

为了从理论上得到三种不同的金属膜的折射 率敏感特性, 我们调节初始共振波长 $\lambda_{\mathrm{R} 0}=650 \mathrm{~nm}$, 此 时对应的测试样品折射率 $n_{4}=1.333$, 之后在不改变 其他条件的情况下, 增加测试样品的折射率至 1.338 , 计算两个折射率下的金属膜的等效折射率 $N_{\mathrm{SP}}$, 通过比较前后两次 $N_{\mathrm{SP}}$ 与 $N_{\mathrm{eff}}$ 交点, 得到由测试样 品折射率增加导致的共振波长的变化量 $\Delta \lambda_{\mathrm{R}}$. 图 6(b) 是对金银合金膜的计算得到的等效折射率 $N_{\mathrm{SP}}$ 和入 射光的等效折射率 $N_{\text {eff }}$ 的色散曲线. 图中显示 $\Delta \lambda_{\mathrm{R}}=$ $30 \mathrm{~nm}$. 采用相同的方法还对金膜和银膜 SPR 传感 器的折射率敏感特性进行了计算, 结果如表 1 所示. 由于初始共振波长均位于 $650 \mathrm{~nm}$ 处, 因此就排除了 由于波长的不同对传感器灵敏度的影响. 从计算结 果可以看出, 金银合金膜 SPR 传感器对于 $\Delta n_{\mathrm{c}}=0.005$
的响应与银膜的响应 $(35 \mathrm{~nm})$ 接近, 波长变化是金膜 计算结果 $(13 \mathrm{~nm})$ 的 2.31 倍. 综合半高峰宽、灵敏度 等因素, 得到的结论是: 金银合金膜 SPR 传感器的 灵敏度较常规的金膜 SPR 传感器提高了 1.31 倍, 而 精确度仅降低了 0.36 倍, 传感器的性能有大幅改 善, 这在下文的实验中可以得到有效的验证. 虽然 计算得到银膜 SPR 传感器的灵敏度和精确度都是 最高的, 但是由于其不稳定性而在实际使用中基本 不被采用.

\section{2 基于三种不同薄膜的 SPR 传感器的折射率灵 敏度测定}

调节测角转盘, 固定入射角 $\theta=11^{\circ}$, 利用不同浓 度的 $\mathrm{NaCl}$ 水溶液作为样品, 对金银合金薄膜 SPR 传 感器的折射率灵敏度进行验证, 得到图 7(a)所示的 实验结果. 图中显示随着 $\mathrm{NaCl}$ 水溶液浓度从 $0 \%$ (质 量分数, 下同)增加到 $10 \%$, 共振吸收峰向长波长方 向有规律的移动. 将图 7(a)中每条光谱曲线的共振 波长的变化量 $\Delta \lambda_{\mathrm{R}}$ ( 以 $0 \%$ 对应的共振波长 $\lambda_{\mathrm{R} 0}$ 为初始 值)对 $\mathrm{NaCl}$ 水溶液的折射率作图, 得到图 7(b)所示的 线性拟合曲线. 拟合曲线的斜率代表传感器的折射 率灵敏度, 从图 7(b)中得到 $\theta=11^{\circ}$ 条件下传感器的灵 敏度 $S=5676.9 \mathrm{~nm} \cdot \mathrm{RIU}^{-1}$.

采用相同的实验方法, 在入射角 $\theta=11^{\circ}$ 条件下对 金膜和银膜 SPR 传感器的折射率敏感特性进行了 测试, 结果如表 2 所示, 在入射角度 $\theta$ 相同的条件下, 基于金银合金薄膜的 SPR 传感器的灵敏度比金膜 SPR 传感器高, 比银膜 SPR 传感器低; 而对于初始共 振波长, 金银合金薄膜 SPR 传感器的 $\lambda_{\mathrm{R} 0}$ 位于金膜和 银膜 SPR 传感器中间, 与仿真计算结果相吻合, 但 实验所测灵敏度 $S$ 普遍低于理论计算结果, 这可能 是由于实验中采用阿贝折射仪对 $\mathrm{NaCl}$ 水溶液折射 率的测定以及通过肉眼读取测角转盘的入射角时 精度不够造成的. 此外, 根据前期研究结果 ${ }^{20}$ 可知, 初始共振波长 $\lambda_{\mathrm{RO}}$ 会影响传感器的灵敏度, 也就是说 在同一实验条件下, $\lambda_{\mathrm{R} 0}$ 越大, $S$ 就越大. 再结合表 2 的 结果, 可以得到如下结论, 在相同的 $\lambda_{\mathrm{R} 0}$ 下, 银膜 SPR 传感器的灵敏度会更高, 金银合金薄膜 SPR 传感器 次之, 金膜 SPR 传感器最低.

实验还在 $\theta=12^{\circ}$ 和 $15^{\circ}$ 条件下进行了测试, 得到 了对应的折射率灵敏度结果, 如图 8 所示. 图 8 中三 条曲线分别代表三种金属膜的测试结果, 在入射角 $\theta$ 相同的情况下, 灵敏度从高到底依次为银膜、金银 合金膜以及金膜 SPR 传感器. 从图中还可以看出随 

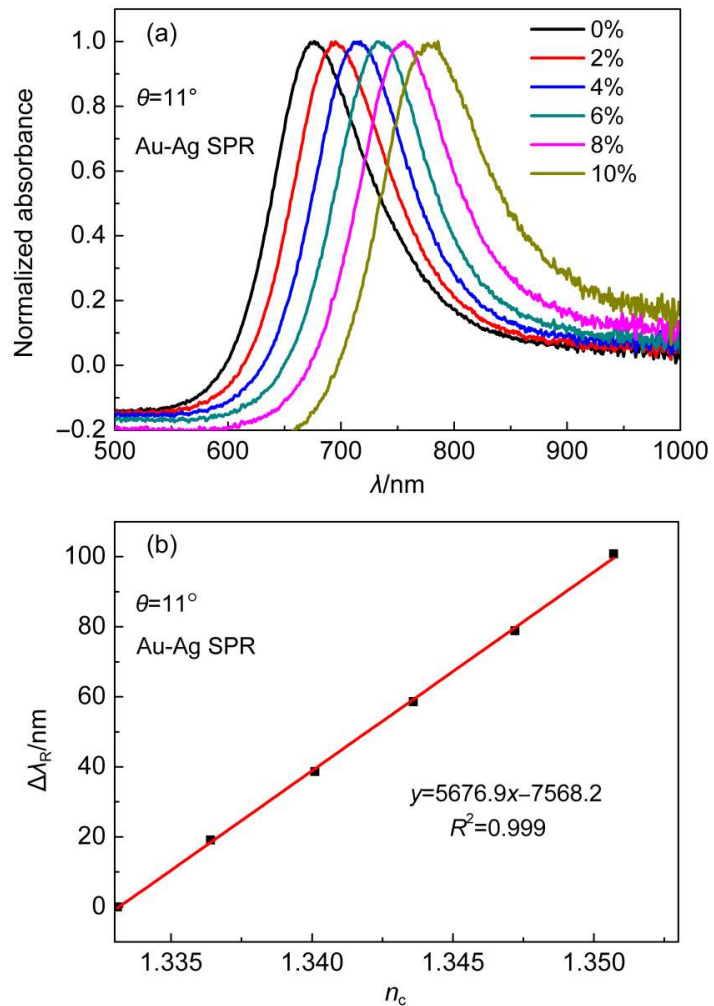

图 7 (a) 在 $\theta=11^{\circ}$ 条件下对不同浓度的 $\mathrm{NaCl}$ 水溶液测得的 金银合金薄膜 SPR 传感器的归一化共振吸收光谱,

(b) $\Delta \lambda_{\mathrm{R}}$ 与 $\boldsymbol{n}_{\mathrm{c}}$ 的线性依赖关系

Fig.7 (a) Normalized resonance absorption spectra of the Au-Ag alloy film based SPR sensor measured with aqueous

$\mathrm{NaCl}$ solutions of different concentrations at $\theta=11^{\circ}$,

(b) linear dependences of $\Delta \lambda_{R}$ with $n_{c}$

着入射角度的增加基于三种金属膜的 SPR 传感器 的灵敏度均下降, 这也与前期研究结果 ${ }^{20}$ 相吻合.

3.3 基于三种不同薄膜的 SPR 传感器的吸附灵敏 度测定

采用阿贝折射仪测定浓度为 $10 \mu \mathrm{mol} \cdot \mathrm{L}^{-1}$ 的 BSA 水溶液的折射率与去离子水相同, 这就意味着 当BSA 水溶液注入到样品槽后, 传感器的响应全部 来源于 SPR 芯片表面吸附了 BSA 分子, 而不是由于 溶液折射率的增加所致. 从前期研究工作可知, ${ }^{20}$

\section{表 2 基于三种金属膜的 SPR 传感器在相同入射角 $\left(\theta=11^{\circ}\right)$ 下初始共振波长和折射率敏感特性 $(S)$ 的对比结果}

Table 2 Comparisons of initial resonance wavelengths and refractive index sensitivities $(S)$ of SPR sensors at $\theta=11^{\circ}$ based on three different metal films

\begin{tabular}{lccccc}
\hline & \multicolumn{2}{c}{ Experimental results } & & \multicolumn{2}{c}{ Simulation results } \\
\cline { 2 - 3 } \cline { 5 - 6 } & $\lambda_{\mathrm{R} o} / \mathrm{nm}\left(\theta=11^{\circ}\right)$ & $S /\left(\mathrm{nm} \cdot \mathrm{RIU}^{-1}\right)$ & $\lambda_{\mathrm{Ro}} / \mathrm{nm}\left(\theta=11^{\circ}\right)$ & $S /\left(\mathrm{nm} \cdot \mathrm{RIU}^{-1}\right)$ \\
\hline Au-Ag SPR & 679 & 5677 & & 677 & 6010 \\
$\mathrm{Au} \mathrm{SPR}$ & 749 & 4540 & & 748 & 4778 \\
$\mathrm{Ag} \mathrm{SPR}$ & 661 & 6595 & & 665 & 7023 \\
\hline
\end{tabular}

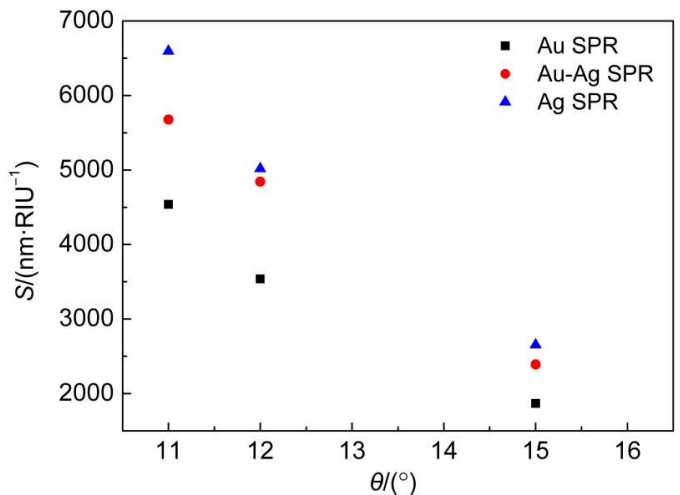

图 8 金银合金薄膜、金膜和银膜 SPR 传感器的折射率 灵敏度与 $\theta$ 的依赖关系

Fig.8 Refractive index sensitivities versus $\theta$ for the Au-Ag alloy film, Au film, and Ag film based SPR sensors

BSA 分子注入样品槽并停留 $15 \min$ 即可在 SPR 芯 片达到吸附平衡. 图 9 所示为采用金银合金膜 SPR 传感器对 BSA 分子的吸附测试得到的结果, 其中黑 色曲线是对去离子水测量得到的归一化共振吸收 光谱, 其初始共振波长 $\lambda_{\mathrm{R} 0}=608 \mathrm{~nm}$, 红色曲线是对 $10 \mu \mathrm{mol} \cdot \mathrm{L}^{-1}$ 的 BSA 水溶液吸附平衡后测得的结果, 其共振波长 $\lambda_{\mathrm{R}}=629 \mathrm{~nm}$, 对比两条曲线可以得到采用 金银合金膜 SPR 传感器测得的共振波长变化量 $\Delta \lambda_{\mathrm{R}}=21 \mathrm{~nm}$.

为了对比, 本文在相同的实验条件下还研究了 BSA 分子在金膜和银膜 SPR 传感器表面的吸附特 性. 由于初始共振波长 $\lambda_{\mathrm{R} 0}$ 会影响传感器的灵敏度, 因此实验中我们调节入射角度 $\theta$ 使得基于三种金属 膜的 SPR 传感器的 $\lambda_{\mathrm{R} 0}$ 近似相等, 测试结果见表 3. 如 表 3 所示, 在几乎相同的 $\lambda_{\mathrm{R} 0}(\sim 610 \mathrm{~nm})$ 下, 金银合金 膜和银膜 SPR 传感器所测得的吸附 BSA 分子引起 的 $\left(\Delta \lambda_{R} \approx 21 \mathrm{~nm}\right)$, 是金膜 $\operatorname{SPR}$ 传感器测试结果 $\left(\Delta \lambda_{R}=7\right.$

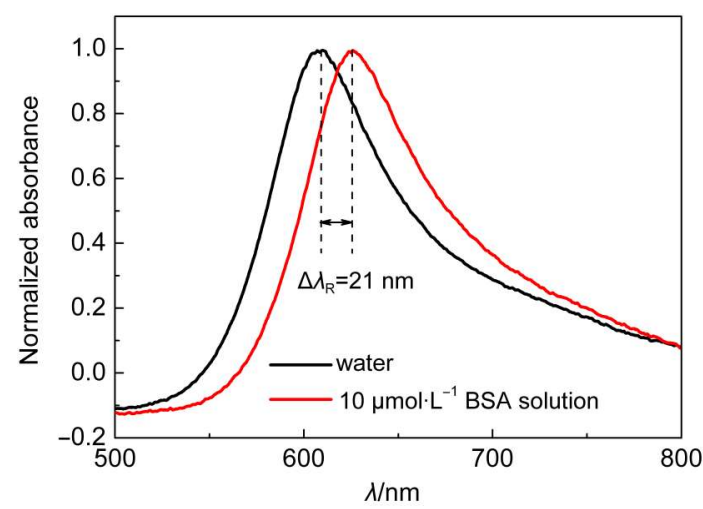

图 9 金银合金膜 $S P R$ 传感器测得的 $10 \mu \mathrm{mol} \cdot \mathrm{L}^{-1} \mathrm{BSA}$ 分子 吸附前后的归一化共振吸收光谱

Fig.9 Normalized resonance absorption spectra of the AuAg alloy film based SPR sensor 


\begin{tabular}{|c|c|c|c|c|}
\hline \multirow{3}{*}{$\begin{array}{r}\text { Table } 3 \\
\text { sensor } \\
\end{array}$} & \multicolumn{4}{|c|}{$\begin{array}{l}\text { Comparisons of adsorption sensitivity of SPR } \\
s \text { at the similar initial resonance wavelength } \\
\text { based on three different metal films }\end{array}$} \\
\hline & \multicolumn{2}{|c|}{ Experimental results } & \multicolumn{2}{|c|}{ Simulation results } \\
\hline & $\lambda_{\mathrm{Ro}}($ water $) / \mathrm{nm}$ & $\Delta \lambda_{\mathrm{R}} / \mathrm{nm}$ & $\lambda_{\mathrm{Ro}}($ water $) / \mathrm{nm}$ & $\Delta \lambda_{\mathrm{R}} / \mathrm{nm}$ \\
\hline $\mathrm{Au}-\mathrm{Ag}$ SPR & 608 & 21 & 607 & 24 \\
\hline $\mathrm{Au}$ SPR & 609 & 7 & 607 & 9 \\
\hline Ag SPR & 610 & 22 & 607 & 24 \\
\hline
\end{tabular}

$\mathrm{nm})$ 的 3 倍. 表 3 还给出了理论仿真结果, 计算时设 定 BSA 吸附层的厚度为 $4 \mathrm{~nm},{ }^{24}$ 折射率为 $1.57,{ }^{25}$ 调 节入射角使得三组仿真中 $\lambda_{\mathrm{R} 0}$ 均为 $607 \mathrm{~nm}$ 不变 $($ 实验 测定与仿真中 $\lambda_{\mathrm{R} 0}$ 的最大差值仅为 $3 \mathrm{~nm}, \lambda_{\mathrm{Ro}}$ 的小幅变 化对计算结果的影响可以忽略), 理论仿真结果与实 验测定数据相吻合, 仿真值略高于实验值. 上述结 果表明, 金银合金膜 SPR 传感器的吸附灵敏度较纯 金膜 SPR 传感器得到了较大的改善, 达到了几乎与 银膜 SPR 传感器相同的水平. 此外, 该金银合金膜 SPR 传感芯片的制作成本较纯金膜 SPR 传感芯片降 低了近一半, 是一种新型的低成本、高灵敏度的检 测芯片, 未来可能会拥有广阔的市场.

\section{4 结 论}

本文研究了基于金银合金膜、金膜以及银膜三 种金属膜结构的 SPR 传感器的敏感特性, 并取得了 实验与理论的详细对比. 利用自建的基于 Kretschmann 结构的波长检测型 SPR 传感器测试平 台, 研究了传感器对 $\mathrm{NaCl}$ 水溶液的折射率灵敏度和 对 BSA 水溶液的吸附灵敏度, 结果表明金银合金膜 SPR 传感器的折射率灵敏度高于金膜 SPR 传感器, 低于银膜 SPR 传感器. 而金银合金膜 SPR 传感器的 吸附灵敏度与银膜 SPR 传感器相近, 是金膜 SPR 传 感器的 3 倍; 理论仿真结果指出金银合金膜 SPR 传 感器的灵敏度与银膜 SPR 传感器接近, 是常规金膜 SPR 传感器的 2.31 倍, 而半高峰宽仅为金膜和银膜 SPR 传感器的 1.36 倍. 综上, 本文提出的金银合金薄 膜 SPR 传感器比常规使用的金膜 SPR 传感器在灵 敏度方面有大幅提高, 而精确度几乎不变, 是一种 高灵敏、低成本、良好稳定性的新型 SPR 传感器.

\section{References}

(1) Homola, J. Chemical Reviews 2008, 108, 462. doi: 10.1021/ cr068107d
(2) Zeng, S.; Baillargeat, D.; Ho, H. P.; Yong, K. T. Chemical Society Reviews 2014, 43, 3426. doi: 10.1039/c3cs60479a

(3) Zijlstra, P.; Paulo, P. M.; Orrit, M. Nature Nanotechnology 2012, 7,379. doi: 10.1038/nnano.2012.51

(4) Zhang, Z.; Lu, D. F.; Qi, Z. M. Acta Phys. -Chim. Sin. 2013, 29, 867. [张 喆, 逯丹凤, 祁志美. 物理化学学报, 2013, 29, 867.] doi: 10.3866/PKU.WHXB201302222

(5) Shalabney, A.; Abdulhalim, I. Optics Letters 2012, 37, 1175. doi: 10.1364/OL.37.001175

(6) Abbas, A.; Linman, M. J.; Cheng, Q. Biosensors and Bioelectronics 2011, 26, 1815. doi: 10.1016/j.bios.2010.09.030

(7) Srivastava, S. K.; Verma, R.; Gupta, B. D. Sensors and Actuators B: Chemical 2011, 153, 194. doi: 10.1016/j. snb.2010.10.038

(8) Linman, M. J.; Abbas, A.; Cheng, Q. Analyst 2010, 135, 2759. doi: $10.1039 / \mathrm{c} 0 \mathrm{an} 00466 \mathrm{a}$

(9) Mitchell, J. Sensors 2010, 10, 7323. doi: 10.3390/s100807323

(10) Hodnik, V.; Anderluh, G. Sensors 2009, 9, 1339.

(11) Zhai, P.; Guo, J.; Xiang, J.; Zhou, F. The Journal of Physical Chemistry C 2007, 111, 981. doi: 10.1021/jp065525d

(12) Frischeisen, J.; Mayr, C.; Reinke, N. A.; Nowy, S.; BrŘtting, W. Optics Express 2008, 16, 18426. doi: 10.1364/OE.16.018426

(13) Mazumdar, S. D.; Hartmann, M.; Kämpfer, P.; Keusgen, M. Biosensors and Bioelectronics 2007, 22, 2040. doi: 10.1016/j. bios.2006.09.004

(14) Qi, Z. M.; Xia, S.; Wei, M.; Matsuda, H.; Zhou, H. Applied Optics 2007, 46, 7963. doi: 10.1364/AO.46.007963

(15) Shankaran, D. R.; Gobi, K. V.; Miura, N. Sensors and Actuators B: Chemical 2007, 121, 158. doi: 10.1016/j.snb.2006.09.014

(16) Hao, P.; Wu, Y. H.; Zhang, P. Acta Phys. Sin. 2010, 59, 6532. [郝 鹏, 吴一辉, 张 平. 物理学报, 2010, 59, 6532.]

(17) Alleyne, C. J.; Kirk, A. G.; McPhedran, R. C.; Nicorovici, N. A. P.; Maystre, D. Optics Express 2007, 15, 8163. doi: 10.1364/ OE.15.008163

(18) Manickam, G.; Gandhiraman, R.; Vijayaraghavan, R. K.; Kerr, L.; Doyle, C.; Williams, D. E.; Daniels, S. Analyst 2012, 137, 5265. doi: $10.1039 / \mathrm{c} 2 \mathrm{an} 35826 \mathrm{c}$

(19) http://www.iri.cnrs.fr/IMG/pdf/SPR.pdf (accessed Mar 20, 2014).

(20) Zhang, Z.; Liu, Q.; Qi, Z. M. Acta Phys. Sin. 2013, 62, 060703. [张 喆, 柳 倩, 祁志美. 物理学报, 2013, 62, 060703.]

(21) Zhang, Y.; Gao, G.; Qian, Q.; Cui, D. Nanoscale Research Letters 2012, 7, 475. doi: 10.1186/1556-276X-7-475

(22) http://www.topac.com/salinity_brix.html (accessed May 12, 2014).

(23) http://en.wikipedia.org/wiki/Fresnel_equations (accessed May $12,2014)$.

(24) Wehmeyer, J. L.; Synowicki, R.; Bizios, R.; García, C. D. Mater. Sci. Eng. C Mater. Biol. Appl. 2010, 30, 277. doi: 10.1016/j. msec.2009.11.002

(25) Chen, H.; Kim, Y. S.; Lee, J.; Yoon, S. J.; Lim, D. S.; Choi, H. J.; Koh, K. Sensors 2007, 7, 2263. doi: 10.3390/s7102263 